\title{
Assessing Completeness of a WEB site from Quality Perspective
}

\author{
J. Sasi Bhanu', DBK Kamesh ${ }^{2}$, JKR Sastry ${ }^{3}$ \\ ${ }^{1}$ CMR Institute of Technology, Kandlakoya Village, Medchal District, Hyderabad, India, \\ ${ }^{2}$ Malla Reddy Engineering College for women Hyderabad, India \\ ${ }^{3}$ Koneru Laksmaiah Education Foundation, India
}

\begin{tabular}{l} 
Article Info \\
\hline Article history: \\
Received Sep 5, 2018 \\
Revised Jul 18, 2019 \\
Accepted Jul 27, 2019 \\
\hline
\end{tabular}

Keywords:

Assessing the quality of web sites

Completeness of the WEB sites

Evaluating the quality of WEB

Quality assessment factors

Total quality of WEB sites

\begin{abstract}
Assessing the quality of a website is most important, especially because people in the world, nowadays, are dependent on the quality of the content hosted on the WEB for various purposes. Every aspect that determines the quality of the website must be taken into account as the users use Websites, for various purposes. The content posted on the WEB should be accessible to the public, and it must be of high quality so that the users can use it without any doubt. The quality of the content posted on the WEB is dependent on many factors. There are as many as 42 factors such as its layout, ease of navigation, structure, quality of content, quality of multimedia, etc. that need to be considered for assessing the overall quality of a website. "Completeness" is one such factor. "Completeness" is about the total availability of the data about a specific context that the user is pursuing. An incomplete website that is not largely used by people eventually gets out of the domain of search engines, and also the users lose interest in such websites. Every quality factor must be assessed independently by using a model. The models must be combined to reflect the overall quality of the website. A set of sub-factors represent a quality factor. The quality of every sub-factor must be individually computed and combined to obtain the quality of the entire factor. This paper primarily focuses on presenting a model, using which, the quality assessment of a website can be made considering the factor called "Completeness" which is one of the factors to be assessed for computing the quality of websites. There can be much data missing in the content posted on the website, such as missing columns in the tables and forms. The missing information makes the website incomplete and un-useful.
\end{abstract}

Copyright $\odot 2019$ Institute of Advanced Engineering and Science. All rights reserved.

\section{Corresponding Author:}

JKR Sastry,

Department of Electronics and Computer Engineering,

Koneru Lakshmaiah Education Foundation (Deemed to be University),

Vaddeswram, Guntur District, Andhra Pradesh, India.

Email: drsastry@kluniversity.in

\section{INTRODUCTION}

Websites are being used extensively for disseminating information. Quality of the websites is the most important and significant issue when it comes to disseminating high-quality content to the users. More refined and relevant information can be made available to the users by considering every aspect determining the quality of the website. It is quite important to determine the factors that influence the quality of the websites by the use of models that help compute the quality of the websites quantitatively to determine the realistic state of the website.

There are more than 42 parameters $[1,2]$ used for computing the quality of the websites. A formulated model required for computing the quality of a Sub-factor. All the individual models need combining into a larger model for computing the quality of the entire factor. 
Exchange of information through web sites has become a part of everybody's life. The content posted on the website is in different formats that include sound, images, videos, audios, graphics, etc. Different formats are used to post different kinds of content. Every day many web sites are being introduced. Quality of these web sites is the real concern. Nobody can make dependent on a content hosted on the WEB in the absence of the quality of such content. Assessment of quality is the most challenging task. Customer satisfaction is dependent on the quality of the website, which is pursued/surfed for want of information.

There is a need to consider many factors for assessing the quality of the websites [3]. The factors include usability, connectedness, structure, navigation, safety, maintainability, reliability, functionality, privacy, portability, etc. There is a need to use many tools, methods, models, and techniques for assessing the quality of a website. Most of the websites developed by organizations require different aspects that include a logo, color scheme, animated graphics, mouse-over effects, graphic art, connectivity with the databases, and several other requisites.

There is a need to use several parameters for evaluating the quality of a website [4], which are either directly related or otherwise. Some of the methods used for computing quality of websites are purely subjective, which are acquired taken as input from individuals. The quality factors can also be measured using statistical methods which are generally known as objective measurements.

Quality requirements differ from stakeholder to stakeholder. Various actors that include The designers, users, management, developers, etc. look at the quality from different perspectives. Maintainability, Security, Functionality, etc. are considered from the perspective of the programmers, while Usability, Efficiency, Creditability, etc. are considered from the perspective of end-users. The needs of a website must be collected first from the perceptive of an actor, and then the quality parameters need to be collected that best represent the said needs.

The complexity of the websites is increasing day by day, especially considering the E-Commerce, Electronics, Museums, and the like websites. Selection of suitable quality factors and determining quality evaluation methods to assess the factors is increasingly complex due to the existence of more factors and the inter-relationships among the parameters. There is a need to consider both attributes and characteristics and the relationship between the two in the websites. Websites must have both objective evaluation and a balanced and conscious subjective inclusion. There is a need to explore both types of methods considering the parameters that need those methods for undertaking the evaluation.

A set of parameters that suit the characteristics and attributes of a website must be selected. Every parameter must be quality assessed such that characteristics and attributes are evaluated hierarchically using the sub-criteria and sub-sub criteria. Evaluating models for each of the characteristics, sub-characteristics, and sub-sub characteristics must be determined, and the same used for computing The quality. There should be a method that combines the quality of the hierarchy of characteristics to determine the overall quality of each attribute/parameter. The overall quality of the website has to be calculated, considering all the attributes that are selected based on the needs examined for the website. In this paper, a model and a process are presented using the quality of the website assessed considering "Completeness" and its related sub-criteria.

\section{LITERATURE SURVEY}

Various factors, such as security, usability, adequacy, and appearance, have been considered, by Miss. Kausar Fiaz Khawaja1 et al., [1] for evaluating the quality of the website. A quality website is easy to use, and it is possible to learn from that kind of a site. One can conclude that the more the website used, the more the quality of the website. The experience of the user could be considered as rich and valuable when the users learn from quality websites. The factor "Appearance" refers to the look and feel of The website which includes appealing, organization of colors and objects, a meaningful arrangement of information, etc. Kausar Fiaz Khawajal et al., [1] have presented a method using which the quality of the websites are computed based on the observations of the users while the website is in use.

Several factors such as Flexibility, safety, and usability have been considered by Vijay Kumar Mantri et al. [3] for computing the quality of the website. The factor "Usability" refers to the effectiveness, satisfaction, and efficient use of the website. The factor "Safety" refers to the presence of the users who is either connected to the website or surfing the website. The user's connection to the website should never get exposed to the outside world. The ability built into the development of a website that allows changes to the website, while the website is in use, is related to the factor "Flexibility." A tool PoDQA (Portal Data Quality Assessment Tool) has been developed using which, one can assess the quality of a website considering factors built into the tool. 
Many factors such as navigation, content, structure, multimedia, appearance, and uniqueness have been considered by Vassilis S. Moustakis et al., [4] for assessing the quality of a website. The factor "content" is related to the information posted on the website and conveyed to the end-user through an interface. The quality factor "content" expresses the extent of generalization and specialization, completeness, connectedness, and coverage of a domain. The support designed and provided to the user that helps to move around the site is called navigation. The quality of navigation is dependent on the effort required to move around the site, availability of the connected links, and simple structure. The quality factor "Structure" deals with speed, organization of content, quick access to the contents, etc. The look and feel of the web site can be related to the appearance and use of different kinds of multi-media and graphics. A website can be designed, considering many styles. A websites "Uniqueness" refers to it standing out among similar websites. A unique website has to be of high quality, and such sites used quite often by the users. Vassilis S. Moustakis et al., [4] have presented a method called AHP (Analytical Hierarchical Process), and they have used the method for computing the quality of the website. Many other parameters also have to be considered for computing the quality of the web site. The issue of "portability" has been considered by Andrina Graniü et al., [5] for assessing the quality of the ability to move content from one site to the other without the need to make any changes at either end.

An assessment system has been presented by Tanya Singh et al. [6] considering different kinds of factors that include appearance, adequacy, security, and privacy. They presented the literal meaning of these factors. The ease of using a website and the extent that one can learn by using the website is to be computed representing the quality of the same in terms of the factor called "usability." The factor "usability, refers to the way the users could use the website. Some information displayed through the website could be private to a user concerned. The eligible Web users provided with the information related to them.

The quality related to the factor "privacy" is the exactness with which the privacy of the usermaintained. The access to the content must be restricted to authorized and authenticated users. The information exchanged between the users and website must be secured so that no damage caused in the transit. The quality of the website can be assessed based on the security enforced during an exchange of information. They have considered another factor "Adequacy" that deals with completeness and exactness of the content hosted on the website. Similar attributes have been considered by Anusha et al., [7] for assessing the quality of the websites. Among the parameters considered by them, the most important attribute is "Portability." The ability to move the code and content from one machine to another without making any change and still make them ready for the target machine is called portability. Another important thing about the websites is the reliability of the content that is displayed to the users every time the user starts surfing the website. A website must reflect the same content every time a user clicks on the same link unless the content on the web site is dynamically changed. The reliability of a website termed as the probability that the intended page is not available.

A website designed and developed such that maintenance of the same is simple when changes to the website are needed. The quality of the web site as such depends on the easiness with which the website maintained. Various issues that include changeability, testability, analysability, etc. must be considered when it comes to assessing the quality of a web site, considering the factor "maintainability." Another important issue considered when it comes to maintainability is the ability to make changes to the website while it is running.

One more important factor considered from the point of quality of the website is the "Analysability" of the website. The analysability of a website includes the ability to read the content, relate the content, and interpret the same, and also find and determine the navigational paths that exist within the website. A website can be said to be stable when no loose ends exist, the user interface is not changed, or the layout of the display is not disorderly presented. The stable website does not undergo frequent changes. The issue of testing a website must be taken into consideration while designing the website. It should be possible to make changes to the websites while the website is in use. Most of the websites being used these days do not support this feature.

Filippo Ricca et al., [8] have considered many other factors which will help to compute the quality of the websites. The factors considered include the design of the website, organization, user-friendliness, and the organization set up of the website. The Organisation of the website includes web pages and the linking of the web pages. The way the web pages are linked have a direct bearing on the effective accessibility of the web pages. The preferences of the users must be kept in mind while designing the websites. The content expected has to be rendered.

Levels to which the content rendered and the playfulness with which the content accessed is the most important factors that must be considered to evaluate the quality of the websites as presented by Saleh Alwahaishi et al., [9]. They have presented the framework but not the computational methods required to compute the quality of the websites. They have presented that a general criterion is required to 
calculate the quality of any website that renders any content and services supported through the website. They have explained that many dimensions considered for computing the quality of the websites. These dimensions must be considered in conjunction with quality indicators and checklists by the WEB designers and developers, as presented by Layla Hasan and Emad Abuelrub [10].

Information dissemination through websites is alarmingly increasing. There has been the rapid growth of web applications and websites. The quality of the websites must be high so that they are used extensively for accessing information needed for different purposes. A model called WebQEM (Web Quality Evaluation Method) has been developed by Kavindra Kumar Singh et al., [11] for computing the quality of websites based on the objective assessment. However, is has been observed that the evaluation of the quality of websites achieved through subjective assessment is most unreliable. They have presented a quantitative assessment of the quality of the website based on objective evaluation. The method proposed by them covers the attributes, characteristics, and sub-characteristics.

Many of the users worldwide are interacting with each other through websites, especially through social web sites. The quality of such social websites has become the most important criteria to facilitate proper interactions among users. Long-Sheng Chen et al. [12] have attempted to find the factors used for evaluating the quality of social sites. They have used feature selection techniques to compute the quality of social sites. Computation of the quality of the websites requires the evolution of Metrics. Naw Lay Wah et al., [13] have presented metrics using which usability of a website computed through sixteen factors that include total pages, word count percentage of body text, text to link count, the total size of the website in bytes, etc. They have used support vectors for predicting the quality of websites.

Sastry JKR et al., [14-18] have presented the way quality assessed considering the attributes which, include content, structure, navigation, multimedia, and usability. The method of considering subcharacteristics and the way the quality assessed relating to an attribute presented. Many other approaches presented relating to the issue of quality of the websites, which lead to newer dimensions to the assessment of the quality of the websites [19-28]. However, none of the papers presented in the literature have dealt with the issue of assessing the quality of the websites from the view of completeness of content hosted on the websites.

\section{INVESTIGATIONS AND FINDINGS}

Completeness of Information hosted on a website is important so that users can see the meaning of the content hosted. Many aspects must be considered relating to the completion of the content hosted on the websites. Missed URL/web pages, missing self-reference hrefs in the same page, Missing Tabular columns in the tables, missing data items in input-output forms, Missing menus as per the context, missing Images, Missing Videos, missing Audios are some of the characteristics of the attribute "Completeness" considered and quality assessed to get the overall quality of the websites.

\subsection{Missed URL/WEB pages}

An URL is said to be missing if a href is present and the corresponding WEB page is absent as per the URL represented directory hierarchy. The quality of these characteristics can be measured based on the number of hrefs missing'. Missing hrefs identified through all the pages developed for implementing the entire website.

Let $\mathrm{n}=$ Number of pages

Total Missing hrefs $=\sum_{1}^{n}$ mherefs $(i)$

Table 1 shows the details of missing hrefs in the code, and the way quality computed considering the number of missing hrefs. This table modified as per the experience gained, considering the perception of the user. Intelligent systems / Expert systems can be built to determine the metric value for the number of missing hrefs.

Table 1. Assessment of quality of Missing hrefs (Qhrefs)

\begin{tabular}{cccccc}
\hline Missing hrefs & 1 & 2 & 3 & 4 & $>5$ \\
\hline Quality factor (Qhrefs) & 1.00 & 0.70 & 0.50 & 0.25 & 0 \\
\hline
\end{tabular}




\subsection{Missing self-referential hrefs}

In an html page, navigation can be implemented using hrefs, especially when the size of the page is too high big or large. The Navigation within the same page implemented through self hrefs. Generally, it is the case that self hrefs coded within the same page, but program control to those hyperlinks are not coded, resulting in the execution of self-reference. Missing self hrefs identified through all the pages developed for implementing the entire website, as shown in Table 2.

Let $\mathrm{n}=$ Number of pages

Total self-Missing hrefs Qshrefs $=\sum_{1}^{n} \operatorname{msherefs}(i)$

Table 2. Assessment of quality of missing self hrefs (Qshrefs)

\begin{tabular}{cccccc}
\hline Missing hrefs & 1 & 2 & 3 & 4 & $>5$ \\
\hline Quality factor (Qshrefs) & 1.00 & 0.70 & 0.50 & 0.25 & 0 \\
\hline
\end{tabular}

\subsection{Missing tabular columns}

Content sometimes is rendered through the display of tables. The columns contained in the tables must match a repository of the data elements that were designed to develop the database. Comparing the html based tables with the existing data repository will help to find whether the tables have been coded properly within the HTML. While comparing the tables, missing columns in the table if any found, the details of the comparison tabulated as per Table 3. The quality of the Tables inserted into the HTML Tables computed as per Table 4 .

Table 3. Missing column details in the tables incorporated into HTML pages

\begin{tabular}{|c|c|c|c|}
\hline Serial number & HTML Page & Number of Tables referred & Number of missing Columns \\
\hline 1 & Kluniversity.in/ecm/reser & 2 & 2 \\
\hline 2 & Kluniversity.in/ecm/programs & 2 & 0 \\
\hline 3 & Kluniversity.in/ecm/publications & 4 & 0 \\
\hline & Total & 8 & 2 \\
\hline
\end{tabular}

Table 4. Quality assessment based on the missing columns

\begin{tabular}{cccccc}
\hline Missing Columns & 0 & 1 & 2 & 3 & $>4$ \\
\hline Quality Factor (Qmiscols) & 1.0 & 0.7 & 0.5 & 0.2 & 0.0 \\
\hline
\end{tabular}

\subsection{Missing fields in forms}

Content may be presented sometimes based on input forms. The attributes reflected as part of forms may be incomplete due to missing of some of the relevant fields designed into displayed forms. The more the fields are missing, the less the quality of the website. Generally, the data to be inputted through the forms has to be in line with the corresponding data repository. Comparing the html based tables with the existing data repository will help to find whether the tables have been coded properly within the HTML. While comparing the tables, missing attributes in the forms if any found, the details of the comparison tabulated as per Table 5 . The quality of the forms inserted into the HTML pages shown in Table 6.

Table 5. Missing field details in the forms incorporated into HTML pages

\begin{tabular}{clcc}
\hline Serial number & \multicolumn{1}{c}{ HTML Page } & Number of forms referred & Number of missing fields \\
\hline 1 & Kluniversity.in/ecm/research & 1 & 0 \\
2 & Kluniversity.in/ecm/programs & 1 & 0 \\
3 & Kluniversity.in/ecm/publications & 2 & 1 \\
& Total & 4 & 1 \\
\hline
\end{tabular}

Table 6. Quality assessment based on the missing fields

\begin{tabular}{cccccc}
\hline Missing fields & 0 & 1 & 2 & 3 & $>4$ \\
\hline Quality Factor (Qmisfields) & 1.0 & 0.7 & 0.5 & 0.2 & 0.0 \\
\hline
\end{tabular}




\subsection{Missing images}

The content rendered on web sites is being enriched from time to time by imbibing multimedia based objects into the content. The quality of the images is an issue. The size of the image and the resolution of the images are some of the major factors. Every HTML page will refer to the images through its URL and generally defines the size and resolution. Sometimes the images as referred by a "href" may not be existing, and as a result, blank images displayed. Secondly, the characteristics of the actual image must match the specification of the image code into the HTML page. Each page can be scanned to determine the number of images contained in it, and then the specification of each of the images in terms of the size and resolution and the location of each of the image can be determined. An image can be retrieved using the href of the image, and the size and resolution of the image can be computed and then compared with code into the HTML page. If the comparison fails or if the image does not exist, then it can be construed as a missing Image - the results obtained through the application of the process explained above shown in Table 7. The quality of the Images inserted into the HTML pages computed as per Table 8. Since there is one missing image and one non-coherent image, the quality computed as 0.75 .

Table 7. Missing images

\begin{tabular}{clcccc}
\hline $\begin{array}{c}\text { Serial } \\
\text { number }\end{array}$ & \multicolumn{1}{c}{ HTML Page } & $\begin{array}{c}\text { Number of } \\
\text { Images }\end{array}$ & $\begin{array}{c}\text { Number of missing } \\
\text { Images }\end{array}$ & $\begin{array}{c}\text { Number of non- } \\
\text { coherent images }\end{array}$ & $\begin{array}{c}\text { Total In-complete } \\
\text { Images }\end{array}$ \\
\hline 1 & Kluniversity.in/ecm/research & 1 & 0 & 0 & 0 \\
2 & Kluniversity.in/ecm/programs & 1 & 1 & 0 & 1 \\
3 & Kluniversity.in/ecm/publications & 2 & 0 & 1 & 1 \\
4 & & $\ldots$ & 4 & 1 & 1 \\
\hline
\end{tabular}

Table 8. Quality assessment based on the missing fields

\begin{tabular}{|c|c|c|c|c|c|}
\hline Missing Images & 0 & 1 & 2 & 3 & $>4$ \\
\hline Quality Factor (QmisImages ${ }_{M}$ ) & 1.0 & 0.7 & 0.5 & 0.2 & 0.0 \\
\hline Non Coherent Images & 0 & 1 & 2 & 3 & $>4$ \\
\hline Quality Factor (QmisImages ${ }_{\mathrm{C}}$ ) & 1.0 & 0.8 & 0.6 & 0.4 & 0.0 \\
\hline Combined quality of Factor (QmisImages) & 1.0 & 0.75 & 0.65 & 0.45 & 0.0 \\
\hline
\end{tabular}

\subsection{Missing videos}

Videos are yet another form of the content included in the websites for providing interactive content. The users normally are quite interested to learn through the Videos posted on the WEB. Availability of videos on the one hand and the quality of video playing, on the other hand, are the two kinds of sub-factors that are seriously concerned with the quality of the website. The videos referenced in an HTML file or a script file through the specification of a URL and the player that must be used locally for playing the videos. The videos referenced through location, length, width, and the number of frames displayed per second. The size of the videos fixed in the code and the actual size of the videos stored at the URL location needs to be matched, failing which compression of videos is undertaken leading to a reduction in the resolution of the video. Availability of videos at the location referenced in the code and matching the specified and stored size of the videos are the two most important criteria for assessing the quality of the videos about the issue of "completeness."

Another Important feature of videos is the mixing of sound into the videos. The videos will be poor quality videos if proper sound mixing has not taken place properly. A video is improper if accompanying sound file does not exist. Thus the quality of video files can be assessed based on loss of accompanying sound files. The resource files of the example site have been scanned to find the number of videos referred could not be located, not coherent considering the mismatch of the referred videos sizes and the actual size of the Videos stored at the locations specified through URLs. Table 9 shows the scanned and processed results.

Table 9. Missing videos

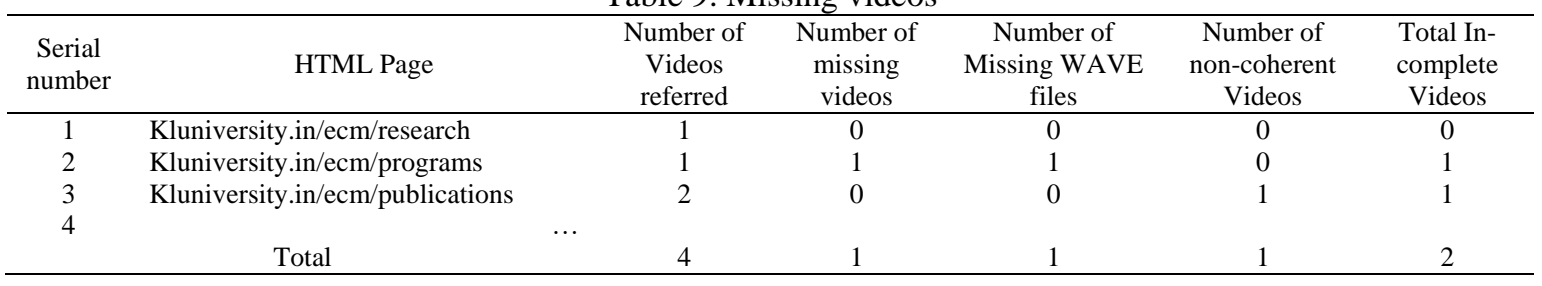


The quality of the Videos inserted into the HTML/JSP pages computed as per Table 10. The quality of videos is computed as 0.766 since there are one missing Video, one non-coherent video, and one missing wave file, the quality of videos computed as 0.766 .

Table 10. Quality assessment based on the missing fields

\begin{tabular}{|c|c|c|c|c|c|}
\hline Missing Images & 0 & 1 & 2 & 3 & $>4$ \\
\hline Quality Factor (QmisVideos $\mathrm{M})$ & 1.0 & 0.7 & 0.5 & 0.2 & 0.0 \\
\hline Non Coherent Videos & 0 & 1 & 2 & 3 & $>4$ \\
\hline Quality Factor (QmisVideosc) & 1.0 & 0.8 & 0.6 & 0.4 & 0.0 \\
\hline Number of Missing WAVE files & 0 & 1 & 2 & 3 & $>4$ \\
\hline Quality Factor (QmisWavew) & 1.0 & 0.8 & 0.6 & 0.4 & 0.0 \\
\hline Combined quality of factor QmisVideos & 1.0 & 0.766 & 0.566 & 0.33 & 0.0 \\
\hline
\end{tabular}

\subsection{Total quality considering the factor "completeness."}

The quality of a web site considering the factor "Completeness" and its attributes computed as an average of the quality of the individual characteristics. The less, the quality of a characteristic, the more the improvement made in respect of that characteristic. The total quality of a website considering the "completeness" factor is dependent on the quality of the related sub-factors. It could be possible that the quality of one sub-factor is high while the quality of some other sub-factor may be quite poor. The sub-factors as such are independent. All the sub-factors are equally important, no weights as such assigned. A simple indication of the quality of the factor could be the average of the quality of all the sub-factors.

Quality of a WEB site (Completeness) = (Qhrefs + Qshrefs + Qmiscols+ Qmisfields + QmisImages + QmisVideos)/6

\section{EXPERIMENTATION AND RESULTS}

The methods presented in this paper applied to an existing website, and the quality of the website computed. Table 11 shows the experimental results. The results reveal that the website can be improved further.

Table 11. Quality assessment of the parameter "completeness."

\begin{tabular}{ll}
\hline Quality Factor & Quality value \\
\hline Qhrefs & 0.700 \\
Qshrefs & 0.700 \\
Qmiscols & 1.000 \\
Qmisfields & 1.000 \\
QmisImages & 0.750 \\
QmisVideos & 0.766 \\
Total & 4.916 \\
Average & 0.819 \\
\hline
\end{tabular}

\section{CONCLUSIONS}

The website must be of high quality such that the users will be interested in the content hosted on the websites. Websites must be designed and developed, considering 42 different quality factors. Quality assessment of each of the factors needs to be done considering sub-factors and the features attached to a factor. An evaluation and assessment model for assessing the quality of a website considering each of the factors separately and later combining the quality of each of the factor for projecting the overall quality of the entire website is needed. A scientific model has to be determined using which, one will be able to assess the quality of a website considering a specific factor.

The quality factor "Completeness" is very important as it reveals the extent to which a website is disconnected. The more disconnected the WEB site, the more it becomes unusable. Users generally lose interest in such kind of web sites. The models proposed in this paper for computing the quality of the websites considering the factor "Completeness" is simple, extendable, and easily implementable. As has been said in the introduction, 42 factors need consideration for evaluating the total quality of the website. Each factor must be modeled separately, considering all the related sub-factors. In the literature, 12 Factors have been modeled and presented. Sastry et al., [2] have presented the details of the remaining factors for researching and finding suitable models which help to compute the quality of the leftover factors. There could be a final attempt to compute the total quality of the website considering the models that have been evolved for computing quality of individual factors separately. 


\section{REFERENCES}

[1] K. F. Khawaja and R. H. Bokhari, "Exploring the Factors Associated With Quality of Website," Department of Technology Management, International Islamic University, Islamabad, Pakistan, vol. 10, pp. 37-45, 2010.

[2] J. K. R. Sastry and T. S. Lalitha, "A framework for assessing the quality of a WEB SITE, PONTE," International Journal of Science and Research, vol. 73, 2017.

[3] V. K. Mantri, "An Introspection of Web Portals Quality Evaluation," International Journal of Advanced Information science and technology, vol. 5, pp. 33-38, 2016.

[4] V S Moustakis, "Website quality assessment criteria," Proceedings of the Ninth International Conference on Information Quality, 2004.

[5] A. Graniü, et al., "Usability Evaluation of Web Portals," Proceedings of the ITI 2008, 30th Int. Conf. on Information Technology Interfaces, 2008.

[6] T. Singh, et al., "E-Commerce Website Quality Assessment based on Usability," Department of Computer Science \& Engineering, Amity University, Uttar Pradesh, Noida, India, pp. 101-105.

[7] R. Anusha, "A Study on Website Quality Models," Department of Information Systems Management, M.O.P Vaishnav College for Women (Autonomous), Chennai, vol. 4, pp. 1-5, 2014.

[8] F. Ricca and P. Tonella, "Analysis and Testing of Web Applications," Centro per la Ricerca Scientifica e Tecnologica, I-38050 Povo (Trento), Italy, 2001.

[9] S. Alwahaishi and V. Snášel, "Assessing the LCC Websites Quality," Springer-Verlag Berlin Heidelberg, in F. Zavoral NDT 2010, SI, CCIS 87, pp. 556-565, 2010.

[10] L. Hasan and E. Abuelrub, "Assessing the Quality of Web Sites," Applied Computing and Informatics, vol. 9, pp. 11-29, 2011.

[11] Singh KK et al., "Implementation of a Model for Websites Quality Evaluation - DU Website," International Journal of Innovations \& Advancement in Computer Science, vol. 3, 2014.

[12] L. S. Chen and P. Chung, "Identifying Crucial Website Quality Factors of Virtual Communities," Proceedings of the International Multi-Conference of Engineers and computer scientists, IMECS, vol. 1, 2010.

[13] N. L. Wah, “An Improved Approach for Web Page Quality Assessment," IEEE Student Conference on Research and Development, 2011.

[14] Sastry J. K. R et al., "Quantifying quality of WEB sites based on content," International Journal of Engineering and Technology, vol. 7, pp. 138-141, 2018.

[15] Sastry J. K. R et al., "Quantifying quality of web sites based on usability," International Journal of Engineering and Technology, vol. 7, pp. 320-322, 2018.

[16] Sastry J. K. R., et al., "Structure-based assessment of the quality of WEB sites," International Journal of Engineering and Technology, vol. 7, pp. 980-983, 2018.

[17] Sastry J. K. R., et al., "Evaluating quality of navigation designed for a WEB site," International Journal of Engineering and Technology, vol. 7, pp. 1004-1007, 2018.

[18] N. P. Kolla, et al., "Assessing the quality of WEB sites based on Multimedia content," International Journal of Engineering and Technology, vol. 7, pp. 1040-1044, 2018.

[19] J. S. Babu, et al., "Optimizing webpage relevancy using page ranking and content-based ranking," International Journal of Engineering and Technology (UAE), vol. 7, pp. 1025-1029, 2018.

[20] K. S. Prasad, et al., "An integrated approach towards vulnerability assessment \& penetration testing for a web application," International Journal of Engineering and Technology (UAE), vol. 7, pp. 431-435, 2018.

[21] M. V. Krishna, et al., "A framework for assessing the quality of a web site," International Journal of Engineering and Technology (UAE), vol. 7, pp. 82-85, 2018.

[22] R. B. Babu, et al., "Analysis on visual design principles of a webpage," International Journal of Engineering and Technology (UAE), vol. 7, pp. 48-50, 2018.

[23] S. S. Pawar and Y. Prasanth, "Multi-Objective Optimization Model for QoS-Enabled Web Service Selection in Service-Based Systems," New Review of Information Networking, vol. 22, pp. 34-53, 2017.

[24] B. Bhavani, et al., "Review on techniques and applications involved in web usage mining," International Journal of Applied Engineering Research, vol. 12, pp. 15994-15998, 2017.

[25] K. K. Durga and V. R. Krishna, "Automatic detection of illegitimate websites with mutual clustering," International Journal of Electrical and Computer Engineering, vol. 6(3), pp. 995-1001, 2016.

[26] T. Y. Satya and Pradeepini G., "Harvesting deep web extractions based on hybrid classification procedures," Asian Journal of Information Technology, vol. 15, pp. 3551-3555, 2016.

[27] S. J. S. Bhanu, et al., "Implementing dynamically evolvable communication with embedded systems through WEB services," International Journal of Electrical and Computer Engineering, vol. 6(1), pp. 381-398, 2016.

[28] L. Prasanna, et al., "Profile-based personalized web search using Greedy Algorithms," ARPN Journal of Engineering and Applied Sciences, vol. 11, pp. 5921-5925, 2016. 